62,73
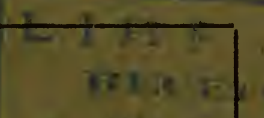

\title{
RETAIL PRICE LIST.
}

CHELTENHAM

i) I

\section{NURSERIES.}

Established 1857.

Oak Lane and Cheltenham Road.

ADDRESS

ROBT. B. HAINES CO.,

Cheltenham P. O., Montgomery Co., Pa. 



\section{RETAIL PRICE LIST}

of

\section{Ornamental and Fruit Trees,}

\section{Evergreens,}

Shrubs, Vines, Bedding Plants, etc. for sale at the

\section{Cheltenham Nurseries,}

Oak Lane and Cheltenham Road.

One mile east from Oak Lane and Elkins Stations (Bethlehem Branch, P. \& R. R. R.).

One mile west from Cheltenham Station (Newtown Branch, P. \& R. R. R.).

P. O. Address, ROB'T. B. HAINES CO.,

Cheltenham P. O., $\quad$ Montgomery Co., Penna.

Freight, Express and Telegraph Address, Oak Lane Station (P. \& R. R. R.), Pa. 
We are glad to welcome visitors to our nurseries any day except Sundays.

Orders by mail will receive prompt and careful attention. All orders should specify clearly how shipments are to be made, whether by freight or express, giving post office as well as shipping address.

Delivery f. o. b. cars at Oak Lane or Cheltenham stations, after which goods are at the risk of the purchaser.

A small extra charge for boxing or baling.

Terms, cash. Orders from strangers should be accompanied by cash or satisfactory references.

We are constantly adding to our stock, and trees or plants not mentioned in this list may be on hand within a short time. Stock not in hand when orders are received will be obtained with the least possible delay and without extra charge. 


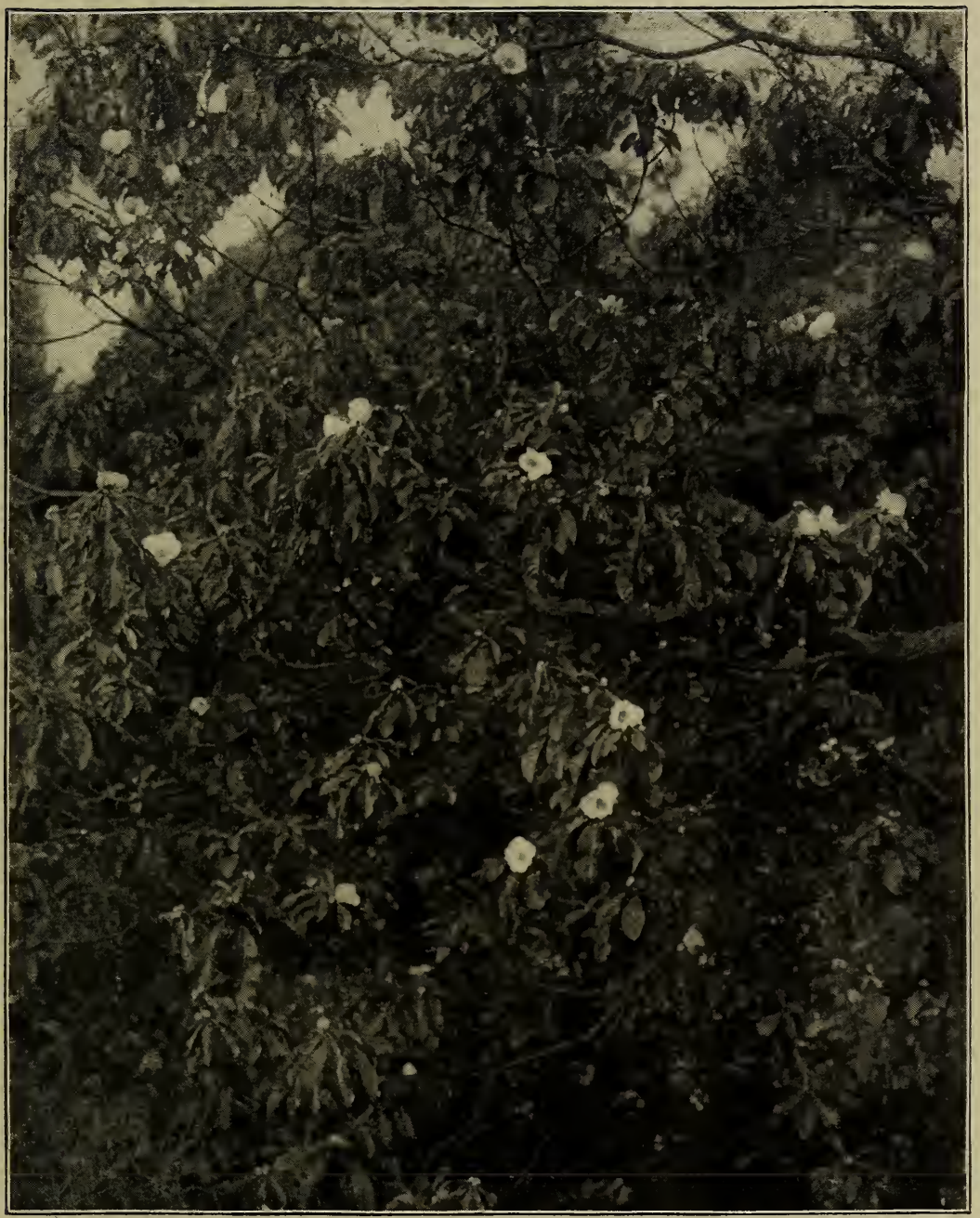

A branch of GORDONIA PUBESCENS. Tree about forty years old, growing at Cheltenham Nurseries. The delicately fragrant flowers, white, with yellow stamens, continue opening from August until frost. The large glossy leaves turn scarlet in autumn. Very rare. 


\section{Ornamental Department.}

\section{Deciduous Trees.}

EACII

Acer dasycarpum Wierii, Wier's cut-leaved Maple...8-10 ft. $\$ 1.00-\$ 1.50$

“ Negundo, Ash-leaved Maple, Box-elder.......8-12 ft. $.75-1.50$

" platanoides, Norway Maple............8-12 ft. $.75-1.50$

" "Schwedleri,Schwedler's purple Maple.8-10 ft. $\quad 1.00-1.50$

" pseudo-platanus, Sycamore Maple..........10-12 ft. $1.00-1.50$

" " " atropurpurea, Purple Syca-

more Maple .................... 8-10 ft. $1.00-1.50$

" rubrum, Red Map!e ...............8-10 ft. $.75-1.00$

" saccharinum. Sugar Maple............8-12 ft. $.75-1.50$

" polymorphum, Japanese Maple, in variety....2-3 ft. 1.00 \& up

Specimen trees of Norway, Sycamore and Sugar

Maples, at special prices.

Aesculus Hippocastanum alba flore plena, Double

white-flowering Horsechestnut ......... 6 ft. $\quad .75-1,00$

Aesculus Hippocastanum rubicunda, Red-flowering

Horsechestnut .................6-8 ft. $.75-1.00$

Amygdalus Persica, Double-flowering Peach....... $.50-\quad .75$

Betula alba laciniata pendula, Cut-leaved Weeping

Birch ....................4-6 ft. $.75-1.00$

Catalpa Bungei, Dwarf Catalpa, Umbrella Tree.....4- 6 ft. $1.00-1.25$

" Speciosa, Western Catalpa ........... 6 ft. $\quad .75$

Cerasus Avium alba plena, Double white-flowering

Cherry ....................6- 8 ft. $\quad .75-1.00$

Cerasus Avium rosea pendula, Weeping pink Japanese

Cherry ......................... 6 ft.

1.25

Cercis Canadensis, American Judas tree........6- 8 ft.

Cornus florida, White-flowering Dogwocd........ 4- $6 \mathrm{ft}$.

"6 flore rubra, Red-flowering Dogwood..3- $4 \mathrm{ft}$.

Cytisus Laburnum, Golden Chain............ 6 ft.

" " Adami, Pink-flowering Laburnum. $4 \mathrm{ft}$.

Fagus ferruginea, American Beech........... $4 \mathrm{ft}$.

" sylvatica, European Beech ........... 5 ft.

" " asplenifolia, Fern-leaved Beech...4- $6 \mathrm{ft}$.

" " $"$ pendula, Weeping Beech.......4-6 ft.

" " Riversi, River's Purple Beech....4- 6 ft.

Fraxinus Americana, White or American Ash...... 8 - $8 \mathrm{ft}$.

" excelsior. European Ash........... $8 \mathrm{ft}$.

Ornus, Flowering Ash ............ 8 - $8 \mathrm{ft}$.

$.75-1.00$

$.75-1.00$

1.50

$.50-1.00$

$.50-\quad .75$

.50

.75

1.25- 2.00

$1.25-1.50$

$1.50-2.00$

Gordonia pubescens, Franklinia ( see cut), prices on application.

Gymnocladus Canadensis, Kentucky Coffee 'Tree....8-10 ft. $1.00-1.50$

Halesia diptera, Silver-bell-large flowered....... 4 - ft. $.75-1.00$

" tetraptera, Silver-bell .............4-8 ft. $.75-1.25$

Koelreuteria paniculata, Varnish Tree.........4-8 ft. $.75-1.25$ 
Larix Europœa, European Larch............. 4 ft. $\quad .50-\quad .75$

“ Leptolepis, Japanese Iarch . ..........4-6 ft. $.75-1.00$

Liquidamber-styraciflua, Sweet Gum..........4-6 ft. $\quad .75-1.00$

Liriodendron Tulipifera, Tulip Tree...........8-10 ft. $\quad .75-1.00$

Magnolia conspicua, White $\ldots \ldots \ldots \ldots \ldots \ldots \ldots \ldots 3 \mathrm{ft} .1 .00-1.50$

"Kobus, White ................ 5 ft. $1.00-1.50$

“ Lennei, Rich Purple ............... $4 \mathrm{ft.} \quad 1.50-2.00$

" purpurea, Purple ................ $4 \mathrm{ft}$. $.75-1.50$

“ Soulangeana, Pink ............... 4 ft. $1.00-1.50$

“ stellata, White Star Magnolia......... 4 ft. $1.50-2.00$

“ tripetala, Umbrella 'Tree................ 8 ft. $.75-1.50$

Morus alba pendula, Teas' Weeping Mulberry......5- $6 \mathrm{ft} . \quad 1.00-1.50$

Paulownia imperialis, Empress Tree...........8-10 ft. $\quad .75-1.00$

Platanus orientalis, Oriental Plane, Buttonwood....8-12 ft. 1.00- 1.50

Populus Carolinensis, Carolina Poplar..........10-12 ft. $\quad .50$

“ Nigra fastigiata, Lombardy Poplar......12-14 ft. $1.00-1.50$

Ptelea trifloliata, Hop Tree................ $5 \mathrm{ft} . \quad .35$

Quercus coccinea, Scarlet Oak.............. s-10 ft. $1.00-1.50$

“ macrocarpa, Mossy-cup Oak..........8-10 ft. $1.00-1.50$

" palustris, Pin Oak .................6- 8 ft. $1.00-1.50$

“ Robur, English Oak................

“ rubra, Red Oak.................... 8-10 ft. $1.00-1.50$

Salisburia adiantifolia, Maidenhair ur Gingko Tree. .8-12 ft. $1.00-1.50$

Salix Babylonica, Weeping Willow ............6-12 ft. $\quad .75-1.50$

“ Americana pendula ...............6.10 ft. $\quad .75-1.50$

Tilia Americana, American Linden, Basswood ......10-i6 ft. .75- 2.00

“ argentea, Silver-leaved Linden ............8-12 ft. $.75-1.50$

“ Europa, European Linden.............. 8-12 ft. $.75-1.50$

Virgilia Lutea (Cladrastis Tinctoria), Yellow-wood..3- $4 \mathrm{ft}$ f. $\quad .75-1.00$

\section{Deciduous Shrubs.}

$2-2 \frac{1}{2} \mathrm{ft}$., 35 cts. each, or $\$ 3.50$ per dozen, except when otherwise stated. Large plants at special prices.

Amygdalus communis, Flowering Almond, pink, white.

Berberis Thunbergii, Japanese Barberry.

vulgaris purpurea, Purple-leaved Barberry.

Calycanthus floridus, Sweet Shrub.

glaucus.

Caryopteris Mastacanthus, Blue Spirea.

Cercis Japonica, Japan Judas ............... $\quad .50-\quad .75$

Chionanthus Virginica, White Fringe............... $\quad .50$

Citrus trifoliata, Hardy Japan Orange.

C'lethra alnifolia, Sweet Pepper Bush.

Crataegus Oxyacantha, Hawthom, pink and white....... $.50-1.00$

Daphne Mezereum, white.

Deutzia crenata plena, Double Deutzia.

“ gracilis, Dwarf Deutzia.

“ Lemoinei. 
Euonymus alatus, Cork-barked Euonymus...........

Exochorda grandiflora, Pearl Bush................

Forsythia suspensa, Drooping Golden Bell.

viridissima, Green-barked Golden Bell.

Hamamelis Virginiana, Witch Hazel.................

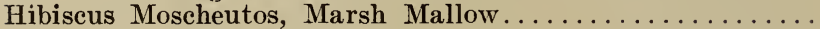

“ Syriacus, Althea, Rose of Sharon ............

Hydrangea paniculata grandiflora, bush form...........

$.25-.35$

$.35-\quad .50$

$.50 \&$ up

" tree form

$.75 \&$ up

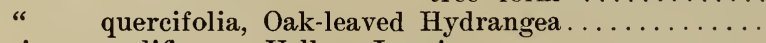

Tasminum nudiflorum, Yellow Jasmine.

Lonicera fragrantissima, Fragrant Honeysuckle.

" Tatarica, Tartarian Honeysuckle.

“ Xylosteum, Fly Honeysuckle.

Pavia parviflora, Dwarf Horsechestnut.............

Philadelphus coronarius, Mock Orange, fragrant.

Pyrus arbutifolia, Chokeberry.

Rhamnus Carolinus, Buckthorn.

Rhodotypus kerrioides, White Kerria

Rhus Copalina, Shining Sumac.

" Cotinus, Mist Tree, Purple Fringe.

"glabra laciniata, Fern-leaved Sumac............

Ribes aureum, Missouri Currant.

Robina hispida, Moss Locust...

Rosa Baby Rambler, Crimson Rambler. Yellow Rambler.

" Dorothy Perkins, Madam Plantier, Jacqueminot.

" Rugosa, red, white.

" Setigera, Prairie Rose.

" rubrifolia, reddish foliage and bark.

"Wichuriana, Memorial Rose, pink. white.

"Several other varieties-prices of all roses..........

Rubus odoratus, Flowering Raspberry.

" sorbifolia, Strawberry-Raspberry ........1 ft.

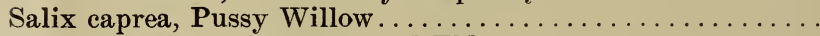

Sambucus aurea, Golden-leaved Flder. . . . . . . . . . . .

" laciniata, Cut-leaved Elder $\ldots \ldots \ldots \ldots \ldots \ldots \ldots$

Spirea Billardi.

" Bumalda.

" " Anthony Waterer

$.35 \&$ up

" prunifolia, Bridal Wreath.

"Reevesiana.

“ sorbifolia, Ash-leaved Spirea.

"Thunbergii.

“Van Houttei.

Stuartia pentagyna $\ldots \ldots \ldots \ldots \ldots \ldots \ldots \ldots \ldots \ldots \ldots$ ft. $.75-1.00$

Styrax Japonica ....................... 4 ft. $.50-1.00$

Syringa, Lilac, white, purple.

Persica, Persian Lilac.

$.35 \& u p$

Tamarix Indica, Tamarisk. 


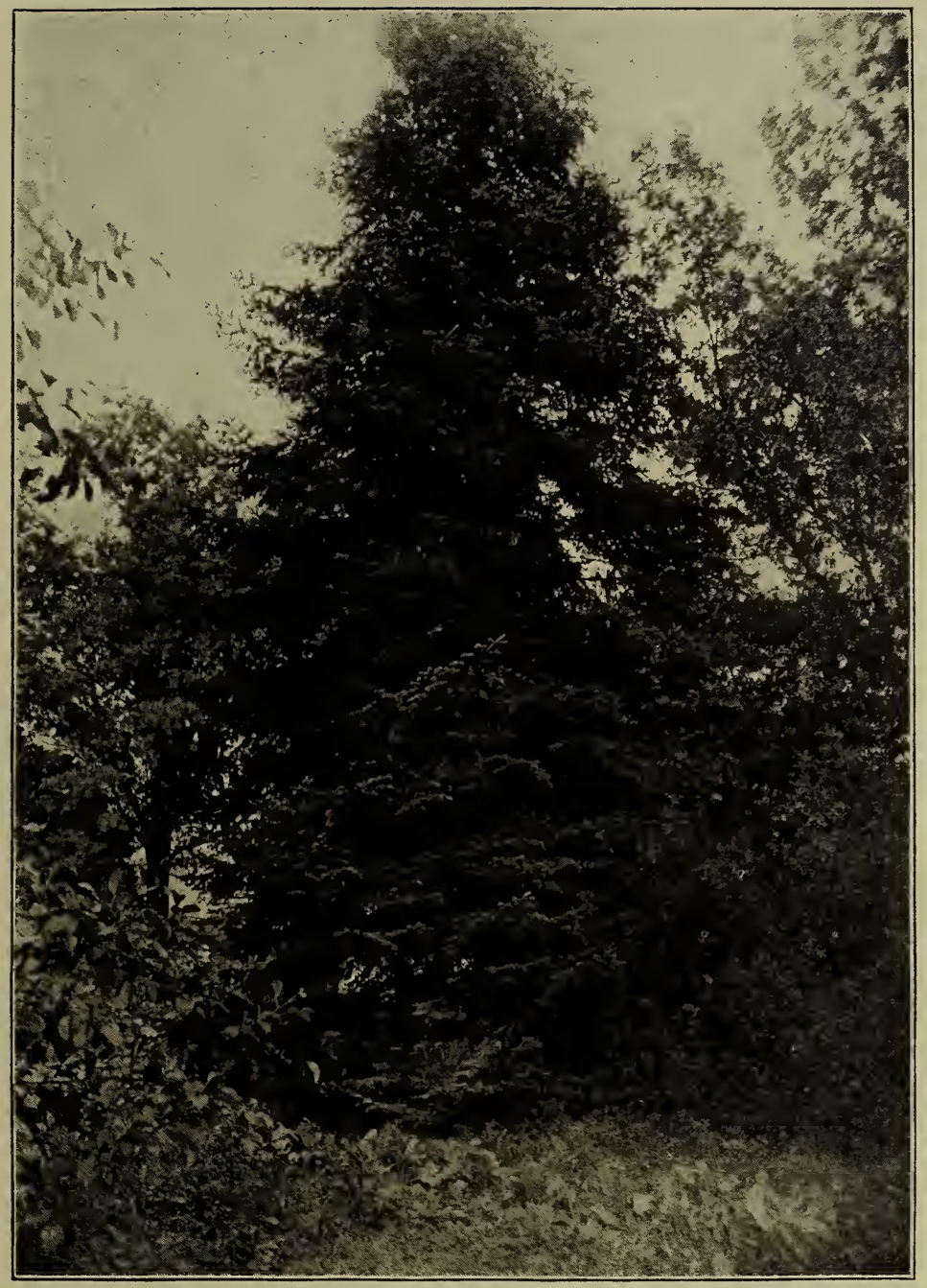

ABIES NORDMANNIANA.

Pin Oak in background. Turkey Oak at right. At Cheltenham Nurseries. 
I'iburnum Opulus sterile, Guelder Rose, Snowball........ . .35- . .

" plicatum, Japanese Snowball.............. .35- ..50

" tomentosum.

Weigela, white, rose, variegated leaved .............. .35- .50

" Eva Rathke.

Xanthoceras sorbifolia $\ldots \ldots \ldots \ldots \ldots \ldots \ldots \ldots \ldots \ldots . \quad .75-1.00$

\section{Vines.}

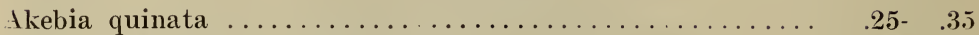

Ampelopsis Veitchii, Japanese Ivy.......................

Hignonia radicans, Common Trumpet Creeper............

" grandiflora, large-flowered Trumpet Creeper........

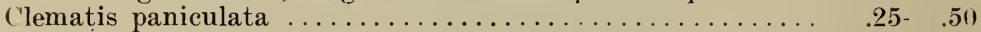

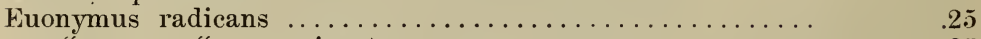

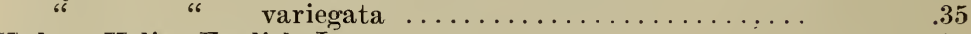

Hedera Helix, English Ivy..................... .25

Lonicera Japonica Halliana, Honeysuckle..............

" "Chinensis, Honeysuckle, purplish foliage .25

" Sempervirens, Coral Honeysuckle . . . . . . . . . . .

Wistaria Chinensis, white, purple $\ldots \ldots \ldots \ldots \ldots \ldots \ldots \ldots, \quad .50-\quad .75$

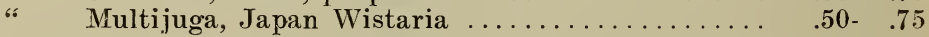

We have also a good assortment of Bedding Plants-annual and perennial-including Columbines, Coreopsis lanceolata, Golden Glow. German, Japan and Spanish Iris, Paeonies. both Tree Peonies and the Herbaceous varieties, Periwinkle, Platycodon, Hardy Phlox, Hardy Ferns, Day Lily, etc., etc.

\section{Evergreens.}

Abies concolor, White Fir .............. 1 ft. $.75-1.00$

" nobilis glauca, Noble Silver Fir.......... $1 \mathrm{ft} . \quad .75-1.00$

" Nordmanniana, Nordmann's Fir ........... $\quad .75-3.00$

Azalea amœna, Evergreen Azalea .............

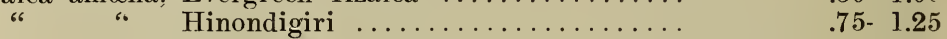

Biota orientalis, Chinese Arborvita $\ldots \ldots \ldots \ldots \ldots .4$ - 4 ft. $\quad .75-1.00$ " " elegantissima, Rollinson's Golden Arborvita

$.75 \&$ up

Buxus arborescens. Tree Box............. $1 \frac{1}{2} \mathrm{ft}$.

$.75-1.00$

.50

.50

Pyramidalis variegata, Prramidal Box....6. 8 in.

.50

" rotundifolia, Broad-leaved Box ........6. 8 in.

100

Cedrus Atlantica Glauca, Mt. Atlas Silver Cedar...2-2 $\frac{1}{2} \mathrm{ft}$. $1.50-2.00$

(Yryptomeria Japonica ....................5- $8 \mathrm{ft} .1 .00 \&$ up

Cupressus Lawsoniana Triomphe de Boskoop, Cypress. 11-2 ft. $\quad .75-1.00$

Ilex crenata, Japanese Holly................1-1 $1 \frac{1}{\mathrm{ft}}$.75- 1.00 
Juniperus Sabina prostrata, Prostrate Juniper . . . $\quad .50-\quad .75$ variegata ................. $1 \mathrm{ft} . \quad .50 \&$ up “Virginiana, Red Cedar ............3- 4 ft. $.75-1.00$ Mahonia aquifolia ..................... $1_{2}^{1} \mathrm{ft.} \quad .50-1.00$ “Japonica ....................1-1 ft. $\quad .35-\quad .50$

Picea alba, White Spruce ............... $3 \mathrm{ft} . \quad 1.50 \&$ up

“ excelsa, Norway Spruce ..............2-5 ft. $. .50-3.00$

“ " inverta. Weeping Norway Spruce.....3- 4 ft. $1.50 \&$ up

" pungens, Colorado Blue Spruce, seedlings.....11 12 ft. 1.25 \& up

“ " Kosteriana, Koster's Blue Spruce... ] ] ft. 1.00 \& up

Pinus Austriaca, Austrian Pine ............. $3 \mathrm{ft} .1 .00$ \& up

" Mugho, Dwarf Mountain Pine.............. $.75 \&$ up

" Strobus, White Pine .................. 5 ft. .75 \& up

“ Sylvestris, Scotch Pine................ 4 ft. 1.00- 1.25

Pseudotsuga Douglasi. Douglas' Spruce...........3- 4 ft. 1.00- 1.50

Retinospora filifera ................... $1 \mathrm{ft} . \quad .50-1.00$

" " aurea ................. l ft. $.50-1.00$

“ obtusa gracilis .................. 5 ft. $1.50-2.00$

“ plumosa ..................... $\quad .50 \&$ ft. up.

" " almea $\ldots \ldots \ldots \ldots \ldots \ldots \ldots \ldots . \ldots \ldots$ ft. up. $.75 \&$ up

“ squarrosa ..................... up. $.75 \&$ ft. up

Rhododendron, various colors $\ldots \ldots \ldots \ldots \ldots \ldots \ldots 1 \frac{1}{2}-2 \mathrm{ft} . \quad 1.25$

Taxus baccata, English Yew ................ l ft. $\quad .75$

" " aurea, Golden Yew............ $1 \mathrm{ft} . \quad .75$

" " fastigiata, Irish Yew.......... $2 \mathrm{ft} .1 .00 \& \mathrm{up}$

Thuya occidentalis, American Arborvita .........2- 6 ft. .50 \& up

$.50 \&$ up

$.50 \&$ up

Thuyopsis borealis, Yellow or Sitka Cypress........1 $1 \frac{1}{2}-2 \mathrm{ft} . \quad 1.00$ \& up

Tsuga Canadensis, Hemlock Spruce.............2-6 ft. $.50 \&$ up

\section{Hedge Plants.}

Prices on application.

Tsuga Canadensis, Hemlock Spruce.

Berberis Thunbergii, Japanese Barberry:

Jigustrum ovalifolium, California Privet.

Retinospora plumosa, Japanese Cedar.

\section{Nuts.}

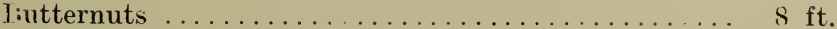

Chestnuts, American .................6. 8 ft.

.

Paragon $\ldots \ldots \ldots \ldots \ldots \ldots \ldots \ldots \ldots \ldots \ldots .6 \mathrm{ft} .1 .00-1.50$

Hazel or Filbert ................... $2 \mathrm{ft} . \quad .50$

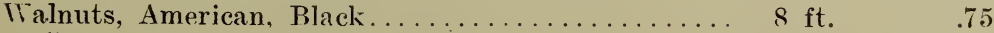

“ English ....................... $6 \mathrm{ft.} .75-1.00$ 


\section{Fruit Department.}

API'LES.

40 cents each and up, according to size.

Early Harvest,

Red Astrachan.

Yellow Transparent,

Fall Pippin, Maiden's Blush, Smoke House, Gravenstein, Duchess of Oldenburg,
Summer.

"

Fall. King

". Northern Spy,

" Smitl's Cider,

" Rhode Island Greening

“ Stayman's Winesap,

Belleflower,

rano,

Grimes Golden,
CRAB APPIES.

Yellow Siberian.

Red Siberian.

PEARS.

60 cents and up, according to size.

Bartlett.

Clapp's Favorite. Keiffer.

Lawrence.

Seckel.

$\checkmark$ ermont Beauty.

And other good varieties.

PEACHES.

25 cents and up, aceording to size.

Early Rivers,

Mountain Rose.

Elberta.

Crawford's Late.

Globe.
Morris White.

Smock.

('hair's Choice.

Tronderful.

Salway.

\section{CHERRIES.}

60 cents and up, according to size.

Dikeman.

Downer's Late.

Elton.

Black Tartarian.
Governor Wood.

May Duke.

English Morello. 
PLUMS.

60 cents and up, according to size.

Green Gage.

Imperial Gage.

Burbank.

QUINCES.

50 cents and up, according to size.

Champion,

Orange,

GRAPES.

25 cents each.

Concord.

Moore's Diamond.

Delaware.

Philadelphia, Herstine,

German Prune.

Damson.

Abundance.

Bourgeat.

Niagara.

Worden.

Clinton.

CURRANTS.

$\$ 1.50$ per dozen.

Fay's Prolific,

Cherry,

Versaillaise.

\section{RASPBERRIES.}

75 cents per dozen.

Golden Queen.

Cuthbert,

Marlborough.

GOOSEBERRIES.

To order. $\$ 1.50$ per dozen.

BLACKBERRIES.

75 cents per dozen.

STRAWRERRIES.

25 cents per dozen.

$\$ 1.50$ per hundred.

RHUBARB.

10 cents per root. $\$ 1.00$ per dozen. 
We hold a Certificate of Inspection from the Department of Agriculture of the State of Pennsylvania, stating that the stock in our Nurseries was duly examined and "found to be apparently free from San José Scale and other dangerously injurious insect pest or pests."

A copy of the certificate will be furnished if desired.

As an additional precaution, stock liable to infestation by San José Scale is fumigated according to directions issued by the Department of Agriculture. 

